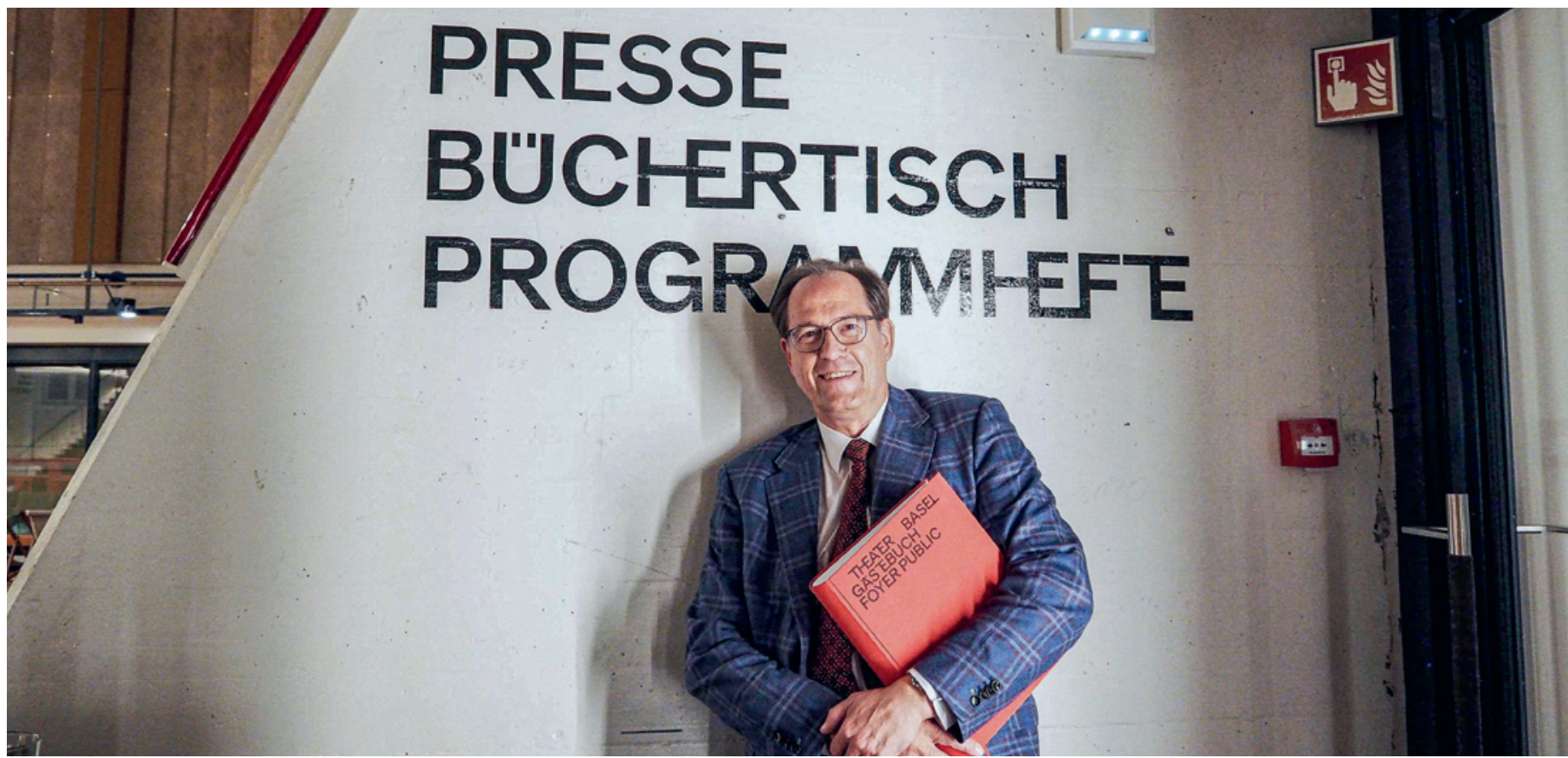

Philipp Tschopp «erbte» das Amt des Theaterarztes bei der Praxisübernahme von seinem Vorgänger.

\title{
Vorhang auf für den Theaterarzt des Theater Basel
}

\section{Nadja Pecinska}

Dr. med., Medizinjournalistin

In dieser Serie werden Medizinerinnen und Mediziner vorgestellt, die aussergewöhnliche Berufsrichtungen eingeschlagen haben. In dieser Ausgabe berichtet Dr. med. Philipp Tschopp über seine Tätigkeit als Theaterarzt am Theater Basel.

Philipp Tschopp, gab es ein bestimmtes Ereignis in Ihrem Leben, das Sie sagen liess: "Ich will zum Theater»?

Nein, eigentlich nicht, es war die Übergabe der Praxis meines Vorgängers an mich, er war 20 Jahre lang Theaterarzt in Basel. Eigentlich wird man nicht Theaterarzt, indem man sagt, «ich will das nun machen», sondern die Aufgabe wird vererbt, wie vom Vater an den Sohn oder von der Mutter an die Tochter. Oder man wird von jemandem, der schon im theaterärztlichen Dienst tätig ist, angefragt, ob man es nicht auch machen möchte.

Wie viel Raum in Ihrem beruflichen Leben nimmt die Arbeit als Theaterarzt ein? Wo sind Sie sonst noch tätig? Ich bin zu 100\% Hausarzt und leite die zwölfköpfige
Gruppenpraxis Socin22 in Basel mit insgesamt vier Ärztinnen und Ärzten. Dann bin ich Verwaltungsratspräsident vom Ärztenetz Nordwest. Und eben Theaterarzt. Ich bin auch die ärztliche Ansprechperson fürs Theater. Letzte Woche haben sie mich angerufen, ein Regisseur komme fast nicht mehr aus seinem Hotelzimmer heraus, ob ich nicht vorbeischauen könnte. Dann rolle ich den roten Teppich aus und mache das. Das ist etwas, was meinen Alltag "aufpfeffert" und mich zusätzlich motiviert. Wenn mir jemand dankt, weil wegen mir die Vorstellung doch stattfinden oder die Filmszene gedreht werden konnte, dann freut mich das. Für den «Fun»-Teil meines Berufes ist der theaterärztliche Dienst für mich wesentlich, ich mache das aus emotionalen Gründen. 
Wie ist der theaterärztliche Dienst organisiert?

Wir sind insgesamt etwa 40 Ärztinnen und Ärzte, die sich alle Vorstellungen teilen. Diese sind wieder in kleinere "Clans» von ca. fünf Leuten unterteilt. Ich zum Beispiel bin immer am Mittwoch im Schauspielhaus und am Freitag auf der grossen Bühne - vorausgesetzt, es findet eine Vorstellung statt. Innerhalb meines «Clans» kann ich dann aber abtauschen, zum Beispiel wenn es sich trifft, dass ich eine Vorstellung schon mehrere Male gesehen habe oder nicht unbedingt ein zweites Mal sehen will - auch das kommt vor. Ich bin im Monat im Schnitt etwa dreimal im Theater, das Maximum ist achtmal; das ist heutzutage aber sehr selten. Früher ist das viel häufiger vorgekommen.

\section{Sind Sie auch bei den Probenprozessen involviert oder nur bei Vorstellungen?}

Wir sind nur bei den Vorstellungen im Einsatz. Denn der Theaterarzt hat ja vor allem zwei Aufgaben, erstens, für die Zuschauerinnen und Zuschauer da zu sein, zweitens, für die Schauspielerinnen und Schauspieler, um alles Erdenkliche möglich zu machen, dass eine Vorstellung läuft.

\section{Sie sehen einige Vorstellungen mehrfach. Wie trotzen Sie der Langeweile?}

Es gibt Vorstellungen, die ich gerne mehrmals sehe. Da ich auch selbst sehr gerne singe, schaue ich mir eine Oper oft ein zweites Mal an. Auch habe ich gemerkt, dass man sich eigentlich jedes Theaterstück zweimal anschauen sollte. Es ist unglaublich, was man alles verpasst, wenn man eine Inszenierung nur einmal sieht. Aber ich muss ehrlich sein, es gab auch schon Abende, da war ich so müde, da habe ich den ersten Akt einfach verschlafen. Dann gehe ich ein zweites Mal, damit ich den ersten Akt auch noch sehe!

\section{Gibt es eine Inszenierung, die Sie nie vergessen werden? Im positiven wie im negativen Sinn?} Ich bin sehr begeistert vom Ballett. Da gibt es nach meinem Geschmack sehr viel Gutes. Ich erinnere mich aber auch an den Il Barbiere di Siviglia von Rossini mit

\footnotetext{
Zur Person

Name: Dr. med. Philipp Tschopp

Alter: 57 Jahre

Am Theater Basel tätig seit: 2004

Funktion: Theaterarzt

Ausbildung:

Allgemeine Innere Medizin,

MSc klinische Epidemiologie
}

Maja Boog, wo sie alle als Insekten auf der Bühne waren, das war sehr originell. Das ist mir geblieben. Oder auch Ibsens John Gabriel Borkman auf der grossen Bühne mit Birgit Minichmayr und Martin Wuttke, eine Koproduktion mit dem Wiener Burgtheater und den Wiener Festwochen. Eine tolle Vorstellung! Manchmal muss ich richtig staunen, was im Theater möglich ist. Der Mehrwert zu Film und Netflix und Co. ist natürlich schon die Dreidimensionalität, direkt dabei sein zu können und auch mal nass zu werden in der ersten Reihe. Mühe habe ich bei experimentellem Theater. Aber es gibt keine Vorstellung, von der ich sage: «Katastrophal!» Ich kann immer etwas mitnehmen. Es ist wie in meiner Sprechstunde: Es ist immer ein Mensch da, den ich wertfrei betrachte. Ich schaue, was bringt er mir, manchmal viel Tolles, manchmal weniger, manchmal muss ich viel geben. Theater ist da ganz ähnlich.

\section{Was ist der häufigste Notfall, den Sie im Theater betreuen müssen?}

Das häufigste ist die vasovagale Synkope, und zwar bei den Zuschauerinnen und Zuschauern. Gerade ältere Leute machen sich einen speziellen Abend, haben vorher vielleicht noch gut gegessen, dann die Wärme des Foyers - plötzlich macht der Kreislauf nicht mehr mit und sie werden ohnmächtig. Es gab mal eine Oper, die startete im Foyer und die Leute mussten eine halbe Stunde stehen. Da waren wir Theaterärztinnen und -ärzte beschäftigt, denn da ist sicher jedes Mal jemand in Ohnmacht gefallen. Normalerweise haben wir aber vielleicht so ein Mal von zehn einen Einsatz, in zwei Dritteln der Fälle bei Zuschauerinnen und Zuschauern.

\section{Was war der dramatischste Unfall, den Sie im Theater erlebt haben?}

Das war vor einigen Jahren in der Oper La Traviata von Giuseppe Verdi. Da gibt es ein grosses Fest, auf dem auch an Champagner nicht gespart wird. Sie hatten auf der Bühne Kunst-Champagner, und eine Flasche ist im wahrsten Sinne des Wortes explodiert. Da fühlte ich mich wie in Beirut, wenn eine Bombe einschlägt. Ich musste den Sängerinnen und Sängern richtiggehend die Splitter aus den Gesichtern herausoperieren. Zum Glück gab es keine Augenverletzungen und alle sind glimpflich davongekommen, die Vorstellung konnte fortgesetzt werden. Wirklich dramatisch aber war die Situation, als eine Schauspielerin Mitten im Stück auf der Bühne einen Herzstillstand hatte. Da kam der Defibrillator zum Einsatz und die Schauspielerin wurde auf der Bühne wiederbelebt. Das Schönste war, als sie die Augen wieder öffnete. Ich habe später vernommen, dass sie wieder spielt, das ist natürlich grossartig! 


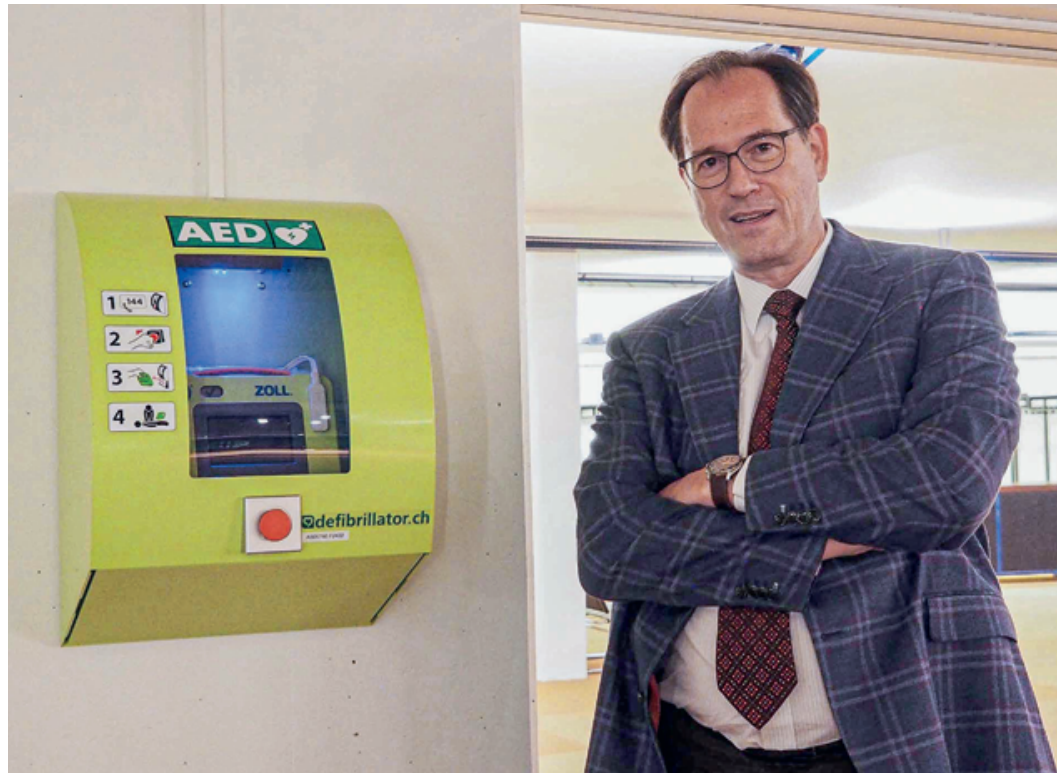

Auch einen Herzstillstand auf der Bühne hat Philipp Tschopp schon einmal erlebt.

\section{Sind Sie schon mal auf eine schauspielerische} Leistung zu Unfall oder Krankheit reingefallen?

Ja, es ist schon einige Male passiert, dass ich schon fast aufgesprungen bin, weil eine Schauspielerin oder ein Sänger hingefallen ist, bis ich merkte, dass es zur Inszenierung gehört.

\section{Wie verändert das Theater Sie als Arzt?}

Das Theater ist eine Bereicherung und für meine WorkLife-Balance sehr wichtig. Es nimmt mich in eine andere Welt, raus aus der sachlichen Sprechstundenrealität. Hier darf ich für einen Moment einfach träumen. Das kann ich mit in den nächsten Tag nehmen, es ist ein emotionaler Mehrwert, etwas, das mich stärkt. Manchmal ist das Theater aber auch wie eine Nachhilfestunde für meine Sprechstunde. Als Hausarzt ist mein Skalpell das Wort, ich muss es genau ansetzen, wie eine Performance im Theater. Ich muss mir überlegen, was für ein Satz kommt, er muss stimmen. Ich schaue also im Theater, wie sich ein Autor in bestimmten Situationen ausdrückt oder wie ein emotionaler Konflikt gelöst wird. Das kann ich dann wiederum in meine Praxis mitnehmen.

\section{Wie viel Theater treffen Sie in der Praxis an?}

Ich sehe meine Arbeit als Hausarzt sehr spielerisch. Die Praxis ist nicht gerade eine Bühne, aber die Leute kommen und haben immer eine Rolle, ich habe meine Rolle, und dann spielen wir. Der Text ist manchmal klar, der heisst Kopfschmerzen, Arbeitsplatzverlust, hoher Blutdruck. Ich kenne diese Stücke auch schon ein bisschen. Dann schaue ich, was inszeniere ich jetzt zu diesem Stück.

\section{Gehen Sie, neben Ihrer Tätigkeit als Theaterarzt, auch sonst noch ins Theater?}

In Basel mache ich das nicht. Privat schaue ich mir an, was auf anderen Bühnen gespielt wird, zum Beispiel mal eine Oper oder eine Ballettvorstellung in Zürich. Da sind alle Tänzerinnen genau gleich gross und genau gleich schlank, das ist ein komplett anderer Ballettstil als hier. Das gefällt mir auch sehr gut.

\section{Ist der Theaterdienst beliebt bei den jüngeren Kolle- ginnen und Kollegen, und wie sieht die Zukunft aus?} Ich muss ehrlich sagen, es ist schon ein zusätzlicher Aufwand. Ich schwinge mich nach der Praxisarbeit auf mein Fahrrad, rase ins Theater, esse nichts, trinke nichts und gehe direkt in die Vorstellung. Also nein, es ist nicht sehr beliebt. Der Wille, etwas Solidarisches zu tun, nimmt meiner Meinung nach generell ab. Wir machen diese Einsätze wirklich für das Theater. Es ist auch kein bezahlter Job, ich bekomme jedes Mal, wenn ich eingeteilt bin, zwei Plätze, kann also jemanden mitnehmen, und that's it; wir Theaterärztinnen und -ärzte sind eigentlich alle Theaterfans.

\section{Sie kennen sicherlich die Ernährungspyramide.} Auf welcher Stufe würden Sie das Theater platzieren? Sicher nicht bei den Kohlenhydraten. Es ist nicht etwas, das man konsumiert und dann macht es noch dick. Ich siedle es zwischen den Genussmitteln und den Vitaminen an. Etwas, das einen aufrechterhält, aber es hat ganz klar auch einen Luxusaspekt. Eigentlich da, wo die Ernährungspyramide aufhört, beim Cognac und den Zigarren!

\section{Und jetzt zum Schluss, ist ein Theaterbesuch gesundheitsfördernd?}

Auf jeden Fall! Das Theater ist einerseits gesellschaftsfördernd, denn meist geht man ja nicht alleine ins Theater, und dadurch friedensstiftend. Andererseits ist das Anschauen und Verarbeiten von Konflikten, das Lachen an sich und das Ausleben von Emotionen unglaublich gesundheitsfördernd. Das Spiel mit der Fantasie ist für uns Menschen eminent wichtig. Man kann an Schönheit teilhaben und sich lebendig fühlen. Theater ist wie Träumen im Wachzustand.

Bildnachweis Nadja Pecinska

Haben Sie als Mediziner auch einen aussergewöhnlichen Beruf den Sie unserer Leserschaft gern vorstellen möchten? Dann freuen wir uns auf Ihr E-Mail an: redaktion.saez[at]emh.ch 